\title{
Required Withholding Period for Vine Leaves Following Spraying with Pesticide
}

\author{
Britt Marianna Maestroni $^{1}$, Iyad Ghanem ${ }^{2}$, Raymond Correll ${ }^{3}$, Amer Abu Alnaser ${ }^{2}$, Marivil Islam ${ }^{1}$, \\ Veronica Cesio $^{4}$, Horacio Heinzen ${ }^{4}$, Andrew Cannavan ${ }^{1}$ \\ ${ }^{1}$ Food and Environmental Protection Laboratory, International Atomic Energy Agency, Vienna, Austria \\ ${ }^{2}$ Department of Molecular Biology and Biotechnology, Syrian Arab Republic Atomic Energy Commission, Damascus, Syria \\ ${ }^{3}$ Biometry Hub, University of Adelaide, Adelaide, Australia \\ ${ }^{4}$ Grupo Analysis Contaminantes Trazas, University of the Republic, Montevideo, Uruguay
}

\section{Email address:}

b.m.maestroni@iaea.org (B. M. Maestroni), eghanem@aec.org.sy (I. Ghanem), rho.environmetrics@bigpond.com (R. Correll), abualnaser@gmail.com (A. A. Alnaser),m.islam@iaea.org (M. Islam), veronicacesio@gmail.com (V.Cesio), heihoracio@gmail.com (H. Heinzen), a.cannavan@iaea.org (A. Cannavan)

\section{To cite this article:}

Britt Marianna Maestroni, Iyad Ghanem, Raymond Correll, Amer Abu Alnaser, Marivil Islam, Veronica Cesio, Horacio Heinzen, Andrew Cannavan. Required Withholding Period for Vine Leaves Following Spraying with Pesticide. Journal of Health and Environmental Research. Vol. 4, No. 4, 2018, pp. 140-152. doi: 10.11648/j.jher.20180404.14

Received: October 25, 2018; Accepted: November 10, 2018; Published: December 17, 2018

\begin{abstract}
Vine leaves are consumed in many countries but little attention is paid to the residues left on them after the application of pesticides that help prevent pests and protect the grapes, the economically important target. Therefore, it is of outmost importance to study the dissipation of the pesticides applied to this crop to protect the consumers that also eat vine leaves. Dissipation kinetics of chlorpyrifos, chlorpyrifos-methyl, diazinon and dimethoate residues were studied in vine leaves grown under sunny conditions in Syria, using an ethyl acetate based sample preparation followed by GC-MS/MS determination. The dissipation rate for all doses applied followed first-order kinetics, with half-lives in grape leaves in the range of 2.9 - 3.9 days. At the recommended application dose, a withholding period of 8.9-37.1 days before consumption should be applied to meet current MRLs and minimise risks to consumers. The effectiveness in the reduction of pesticide loads in vine leaves through washing with either cold or hot water was dependant on the physicochemical properties of the studied pesticides. Hot water washing was very effective for dimethoate, a polar and water-soluble pesticide, with an effective reduction of $92 \%$ of the residue level; but no significant effect was observed for chlorpyrifos, the most apolar compound in this study.
\end{abstract}

Keywords: Vine Leaves, Dissipation Kinetics, Half-Life, Withholding Period

\section{Introduction}

Grape (Vitis vinifera) was estimated by Food and Agriculture Organisation of the United Nations to be the most widely cultivated fruit crop in the world [1]. The Mediterranean countries constitute the main area of cultivation of grapevines. While the main product are grapes, with most of the production destined for wine making, vine leaves are also important for cultural and nutritional reasons. Vine leaves have been used as a nutritious food in Greece and the Middle East for centuries and their popularity as a healthy food is increasing globally [2]. In countries such as
Syria, where vine leaves are widely consumed, it is important to assess human dietary exposure to residues of pesticides applied to vine leaves. As vine leaves may represent an important contribution to the Mediterranean diet, data on the fate of pesticides in vine leaves after application are essential for both the calculation of the theoretical maximum daily intake and the establishment of the Maximum Residues Limits (MRLs) [3]. Most studies on pesticide residues deal with the analysis of grapes or the transformation from vine to wine, as discussed in the review by Grimalt et al. [4]. To date only a few studies have been dedicated to residues in vine leaves and foliage in general [5-10]. Insecticides and 
fungicides are applied to be effective against a wide range of insect pests, such as grape moth (Lobesia botrana), and fungal diseases such as downy mildew (Plasmopora viticola), powdery mildew (Uncinula necator) and gray mold (Botrytis cinerea), but their use has to be strictly regulated. The legal parameter with respect to pesticide residues, which determines whether a food product may be placed on the market, is the MRL expressed in $\mathrm{mg} / \mathrm{kg}$ of product. This value is calculated from toxicological data such as Acceptable Daily Intake (ADI in $\mathrm{mg} / \mathrm{kg} /$ day) and Food Daily Intake (FDI in $\mathrm{kg} /$ day), and agronomic data such as active dose and dissipation curves in the field FAO and the World Health Organization (WHO), through their Joint Meeting on Pesticide Residues (JMPR) meetings evaluate pesticides and their residues in food to set MRLs for food commodities [1112]. The occurrence of pesticides in the environment, as well as in vine leaves, remains an important challenge to address in Syria and elsewhere. The agricultural practices, including pesticide management practices, must be continually improved to meet it. It is important to generate analytical data on the actual levels of crop protection chemicals on crops and in the environment, and to consider these data with respect to the agricultural practices applied under local conditions to be able to optimize pesticide management. In assessing the impact of dietary exposure to pesticides, a number of parameters have to be taken into account and carefully evaluated, including MRLs, withholding times and dissipation rates and post-harvest management, amongst others. These parameters are obtained either experimentally or through modelling and they vary according to the pesticide, the type of crop and the prevailing environmental conditions. A combination of effects results in a reduction, over time, from the initial amount of active ingredient on crops after application. The dissipation rate $\left(\mathrm{K}_{\text {diss }}\right)$ is an important kinetic parameter used to calculate residual concentrations of pesticides in crops harvested for human or animal consumption [13]. The term dissipation is defined as an integrated process where several effects play a role, including volatilization, photodegradation, wash-off, leaching, hydrolysis, chemical and biological degradation, among others [13]. Dissipation rates are usually expressed as the pesticide half-life; the half-life being the time required for the pesticide residue level to fall to half of the initial concentration directly after application. Because of the large combinations of pesticides and crops, it is very important to estimate dissipation half-lives to contribute to risk assessments, for example when establishing MRLs for pesticides in various commodities. The dissipation rate for pesticides applied on a specific crop depends on several factors: the chemical formulation and application method, climatic conditions - especially rainfall and temperature, vapour pressure of the pesticides, and the potential for photodegradation and other chemical degradation [14]. This means that dissipation curves are very specific to local conditions in each growing area [15-20], and therefore generation of precise knowledge of pesticide degradation kinetics is very important to produce reliable data for international bodies, such as Codex Alimentarius Commission, that set MRLs [21]. Because of the agronomic behaviour and differences among regions in the world, the same active ingredients may have different MRLs depending on the country and the climatic conditions [22]. Fantke et al. [13] systematically reviewed 811 scientific literature sources, and analysed 4513 dissipation half-lives of 346 pesticides measured in 183 plants. The authors emphasized that further experiments are needed to analyse pesticide-plant species combinations that so far have not been covered and to allow the use of prediction models. The fundamental reason is because modelling dissipation in plants is highly uncertain and the estimation of dissipation half-lives strongly relies on experimental field data. A study was initiated in Syria on the behaviour of specific organophosphate pesticides (OP) in the field under the current agricultural practices for grape vines. The compounds included in the study were chlorpyriphos, chlorpyriphos methyl, diazinon and dimethoate. OPs are extensively used due to their high insecticidal activity and relatively low persistence [5]. The dissipation rate of OPs is very fast [23-25]. The objective of the study was to establish the withholding period (WHP) for these specific OP pesticides used in vineyards in Syria. The WHP is the minimum period of time that must be allowed after pesticide application before the treated area or crop can be grazed, cut for fodder or harvested. Withholding periods vary for different pesticide/crop combinations. They help to ensure that residues in the treated crop will not exceed the maximum residue limits when the crop is placed on the market [26]. When being prepared as a food in the home (or by industry), vine leaves are first washed. The fate of pesticide residues on raw agricultural crops has been well studied and recently well reviewed by Amvrazi [27], Kaushic [28], Holland [29]. According to these studies the rinsability of a pesticide is not always correlated with its water solubility and different pesticides may be rinsed off commodities using different washing procedures and washing agents [27]. In the present study, after sampling, vine leaves were analysed unwashed, washed with cold water, and washed with boiling tap water to assess whether domestic washing can offer a practical method for decreasing the intake of pesticides for consumers.

\section{Materials and Methods}

\subsection{Field Trials}

\subsubsection{Site Descriptions}

The experimental trials were carried out during the summer of 2016 in two different vineyards located outside the city of Damascus, Syria. A local cultivar of table grape was used for testing. The Al Soujah (Yafour) area, located 23 $\mathrm{km}$ west of Damascus, is an arid zone where the vineyard is surrounded by olive trees and is an experimental station belonging to the Atomic Energy Commission of Syria (AECS). The Al-Hamah area is a private vineyard in a greener zone located $14 \mathrm{~km}$ north west of Damascus and close to a river (see Figures $A 1$ and $A 2$ in the Appendix). 
Meteorological data were collected by an agrometeorological station in Damascus. During the field trials the average daily temperature was $26^{\circ} \mathrm{C}$, the maximum temperature was $36^{\circ} \mathrm{C}$, the average humidity was $27 \%$, the average solar radiation was $209 \mathrm{w} / \mathrm{m}^{2}$, the average wind speed was $11.1 \mathrm{~km} / \mathrm{h}$ and there was no rain. Each growing area was divided into two zones. One zone was treated and the other one was left untreated to be used as a control. The vines did not receive any irrigation, or pesticide or fertilizer treatment throughout the growing season. Four different formulations of organophosphate pesticides (OPs) were purchased from a local supplier and applied at the concentrations indicated on the labels, using a $20 \mathrm{~L}$ hand-pump pesticide sprayer (in $\mathrm{Al}$ Soujah area) and an electronic sprayer (in Al-Hamah area). Care was taken to ensure that the vines were well covered with the spraying mixture. The formulations applied to the vine areas, their label concentrations and the target application rate for each pesticide are presented in Table 1. Formulated forms of the pesticides dimethoate $(400 \mathrm{mg} / \mathrm{mL})$ and chlorpyrifos ethyl $(480 \mathrm{mg} / \mathrm{mL})$ were from AgriPest, diazinon $600 \mathrm{mg} / \mathrm{mL}$ was from Orient for Veterinary Pesticides and chlorpyrifos methyl $(20 \%, 200 \mathrm{mg} / \mathrm{mL})$ was from OvaGreen. At the Al Soujah site, $50 \mathrm{~m}^{2}$ of vines trees were treated with $7 \mathrm{~L}$ of the pesticide formulation mixture, at the Al-Hamah site $100 \mathrm{~m}^{2}$ of vines were sprayed with $100 \mathrm{~L}$ of the formulation mixture. In both cases the total volume of prepared pesticides was sprayed on the vines to deliver the amount of $\mathrm{kg} / \mathrm{Ha}$ as shown in Table 1 . Only one pesticide application was carried out at both locations at the end of the flowering stage.

Table 1. OP insecticides, their concentration and the amount sprayed on vine leaves in Al-Hamah and Al Soujah area.

\begin{tabular}{|c|c|c|c|c|c|}
\hline Insecticide & $\begin{array}{l}\text { Label concentration } \\
(\mathrm{mg} / \mathrm{mL})\end{array}$ & $\begin{array}{l}\text { Amount of OP used for the } \\
\text { mixture in Al Hamah (mL) }\end{array}$ & $\begin{array}{l}\text { Amount sprayed in } \\
\text { Al Hamah (kg/Ha) }\end{array}$ & $\begin{array}{l}\text { Amount of OP used for the } \\
\text { mixture in Al Soujah (mL) }\end{array}$ & $\begin{array}{l}\text { Amount sprayed in } \\
\text { Al Soujah (kg/Ha) }\end{array}$ \\
\hline Dimethoate & 400 & 50 & 2 & 7 & 0.56 \\
\hline Diazinon & 600 & 50 & 3 & 7 & 0.84 \\
\hline Chlorpyrifos-ethyl & 480 & 50 & 2.4 & 7 & 0.672 \\
\hline
\end{tabular}

\subsubsection{Sampling}

For studying the dissipation of the four evaluated OP pesticides, vine leaves were collected at time 0 (one hour after spraying, when the spraying mixture had dried) and then after 1, 7, 14 and 21 days. Sufficient leaves were randomly collected from at least 5 vine trees to provide an overall sample weight of $500 \mathrm{~g}$ of vine leaves. The samples were labelled and immediately dispatched to the laboratory, where they were frozen and kept at $-18^{\circ} \mathrm{C}$ until analysis.

\subsection{Chemicals}

All chemical analyses were performed at the Joint FAO/IAEA laboratories in Seibersdorf, Austria. All water used in this study was obtained from an in-house purification system (Milli Q, Millipore, USA). Certified pesticide analytical standards and triphenylphosphate (TPP, certified analytical standard) were purchased from Sigma Aldrich, Austria. Stock solutions of individual standards were prepared at $10 \mathrm{mg} / \mathrm{mL}$ and used in the preparation of a mixed stock solution at $25 \mathrm{ng} / \mu \mathrm{L}$. The individual stock solution and the stock mixture were stored in amber screw-capped vials with septa in the dark at $-20^{\circ} \mathrm{C}$. Working standard solutions were prepared from the mixed stock solution according to requirements. Residue grade glacial acetic acid and ethyl acetate were purchased from Merck (Austria) and ascorbic acid was purchased from Sigma Aldrich (Austria). Acidified ethyl acetate was prepared by dissolving ascorbic acid $(0.5 \mathrm{~g})$ in milliQ water $(10 \mathrm{~mL})$, to which glacial acetic acid $(10 \mathrm{~mL})$ was added and the volume made up to $1 \mathrm{~L}$ with ethyl acetate. The solution was stored in a screw-capped bottle with septum, wrapped with aluminium foil and kept in the dark at $4^{\circ} \mathrm{C}$. Residue grade sodium hydrogen carbonate $\left(\mathrm{NaHCO}_{3}\right)$, anhydrous sodium sulphate $\left(\mathrm{NaSO}_{4}\right)$ and anhydrous magnesium sulphate $\left(\mathrm{MgSO}_{4}\right)$ were purchased from Sigma Aldrich (Austria). Residue grade primary-secondary amine (PSA) sorbent was purchased from Varian (USA).

\subsection{Sample Preparation}

Upon arrival at the laboratory each sample was divided into three parts. One part was directly stored at $-20^{\circ} \mathrm{C}$ pending analysis. The other two parts were washed by immersion for 5 seconds into either boiling water or tap water, air dried in the dark and stored $-20^{\circ} \mathrm{C}$ pending analysis. Before analysis, the vine leaves were prepared by removing the stems and crushing the leaves to small particles using liquid nitrogen in a mortar and pestle. To ensure effective homogenization, the samples were further homogenized, while still frozen, using a common food chopper (Moulinex, $1000 \mathrm{~W}$ ) for 1 minute.

\subsubsection{Analytical Method}

A validated method for the determination of chlorpyrifos, chlorpyrifos-methyl, diazinon, and dimethoate was applied for the analysis of the samples [30]. Analytical portions of 2 $\mathrm{g}$ of homogenised vine leaves were weighed into $50 \mathrm{~mL}$ labelled PTFE centrifuge tubes. Milli Q water $(4 \mathrm{~mL})$ was added and the samples left to soak for $30 \mathrm{~min}$. Acidified ethyl acetate $(4 \mathrm{~mL})$ and the surrogate standard, TPP $(200 \mu \mathrm{L}$ of a $2 \mathrm{ng} / \mu \mathrm{L}$ solution) were added to each sample. The tubes were shaken vigorously by hand for 1 minute and then for $30 \mathrm{~min}$ on a horizontal shaker. After this step the first salt mixture $\left(0.3334 \mathrm{~g} \mathrm{NaHCO}_{3}+2 \mathrm{~g} \mathrm{NaSO}_{4}\right)$ was added, the tubes were hand-shaken vigorously and the contents were thoroughly homogenized using an ultra-turrax homogenizer (IKA-T25, IKA, Germany) at maximum speed for $1 \mathrm{~min}$. The homogenates were centrifuged for $5 \mathrm{~min}$ at approximately $12,600 \mathrm{~g}$ at $20^{\circ} \mathrm{C}$. Two $\mathrm{mL}$ of the organic supernatant were 
cleaned up in an Eppendorf tube of $15 \mathrm{~mL}$ by adding a second salt mixture (50 mg PSA $+300 \mathrm{mg} \mathrm{MgSO}_{4}$ ), and vortexed for 30 seconds. They were then more intensively agitated on an orbital vortex mixer (Scientific industries) at maximum speed for $5 \mathrm{~min}$. The tubes were then centrifuged for $5 \mathrm{~min}$ at approximately $12,600 \mathrm{~g}$ at $20^{\circ} \mathrm{C}$. The extracts were filtered using a $0.2 \mu \mathrm{m}$ syringe filter yielding a $0.5 \mathrm{~g}$ $/ \mathrm{mL}$ sample extract. Finally, $100 \mu \mathrm{L}$ of internal standard (sulfotep, $1 \mathrm{ng} / \mu \mathrm{L}$ ) was added to $900 \mu \mathrm{L}$ of each extract, including calibration standards, to reach a final volume of 1 $\mathrm{mL}$ and injected into the GC-MS/MS triple quadrupole system.

\subsubsection{Apparatus and Analytical Conditions}

The gas chromatography system used to analyse the pesticide residues consisted of an Agilent 7693 autosampler, 7890 B GC and 7000C GC-MS/MS triple quadrupole system (Agilent Technologies, Santa Clara, USA). Data acquisition and processing were implemented using Agilent Mass Hunter Quantitative Analysis B07.00 software. Two $15.0 \mathrm{~m}$ x 0.25 mm ID x $0.25 \mu \mathrm{m}$ HP-5 ms ultra-inert columns (Agilent $\mathrm{J} \& \mathrm{~W}$, USA) were connected by an electronic pressure controller to enable a 5-minute post-run backflush. The samples were injected using a multimode injector inlet in splitless mode through an ultra-inert inlet liner with a glass wool plug (Agilent Technologies, Santa Clara, USA). The injection volume was $3 \mu \mathrm{L}$. The temperature programmable injector port was set up to an initial injection temperature of $70^{\circ} \mathrm{C}$ for 0.02 minutes and then ramped up to $320^{\circ} \mathrm{C}$ (at $850^{\circ} \mathrm{C} / \mathrm{min}$ ) for 5 minutes, then cooled down to $280^{\circ} \mathrm{C}$ at $20^{\circ} \mathrm{C} / \mathrm{min}$. The oven temperature was held at $70^{\circ} \mathrm{C}$ for 1 minute, then ramped to $150^{\circ} \mathrm{C}$ (at a rate of $50^{\circ} \mathrm{C} / \mathrm{min}$ ), increased to $200^{\circ} \mathrm{C}$ at $6^{\circ} \mathrm{C} / \mathrm{min}$ followed by a final ramp to $280^{\circ} \mathrm{C}$ at $16^{\circ} \mathrm{C} / \mathrm{min}$. The total run time was $24 \mathrm{~min}$. Helium
(99.999\% purity) was used as the carrier gas and nitrogen (99.999\% purity) as the collision gas. The transfer line was maintained at $280^{\circ} \mathrm{C}$. The retention time lock setting (RTL) used chlorpyrifos-methyl as the locking compound at retention time of $10.83 \mathrm{~min}$. The instrument worked at a constant flow $(1.25 \mathrm{~mL} / \mathrm{min}$ in column 1 and $1.45 \mathrm{~mL} / \mathrm{min}$ in column 2). The ion source and the quadrupole analyser temperatures were fixed at $300^{\circ} \mathrm{C} / \mathrm{min}$ and $150{ }^{\circ} \mathrm{C} / \mathrm{min}$, respectively. The transitions and the collision energies used for detection are shown in the Appendix (Table Al of the Appendix). A twenty-five time-segment method was created to obtain adequate sensitivity, and in each time segment dwell times were optimised to collect at least 12 points across a peak (cycles between 3 and 4). The solvent delay was 4 minutes.

\subsubsection{Residues Analytical Determination}

The analytical calibration was carried out using matrix matched standards calibration curves, covering the range $0.005-0.240 \mu \mathrm{g} / \mathrm{g}(2.5-120 \mu \mathrm{g} / \mathrm{L})$ and using the bracketing calibration modality. When needed, the samples and the corresponding blanks, used for the preparation of the matrix matched calibration curves, were diluted with acidified ethyl acetate to meet the analytical range of $0.005-0.240 \mu \mathrm{g} / \mathrm{g}$. Where more than a single estimate of the residue level was available (based on different dilutions required for assessment) an average estimate was used for that sample. The limit of quantitation (LOQ) and the precision data obtained during method validation are presented in the Appendix (Table A2 of the Appendix). Current EU and Codex MRLs for chlorpyrifos, chlorpyrifos-methyl, diazinon and dimethoate are given in Table 2. Values below the minimum reporting level or lowest calibrated level (LCL) were not included in the data set.

Table 2. European Union MRL for grape leaves and current Codex MRL for commodities similar to vine leaves, and selected properties of the three OP pesticides (from Lewis et al. [31]).

\begin{tabular}{llllll}
\hline Pesticide & MRL-EU (mg/kg) & $\begin{array}{l}\text { MRL-Codex Alimentarius (mg/kg) for } \\
\text { similar commodities }\end{array}$ & Koc & $\begin{array}{l}\text { Kow } \\
\text { palf-life on } \\
\text { plants (days) }\end{array}$ & $\begin{array}{l}\text { Photolysis half-life } \\
\text { (days) }\end{array}$ \\
\hline Chlorpyrifos & 0.05 & 0.5 (grapes) & 8151 & 50100 & 3.3 \\
Chlorpyrifos-methyl & 0.05 & 0.5 (grapes) & 4645 & 10000 & 2.4 \\
Diazinon & 0.01 & 0.5 (lettuce leaf) & 609 & 4900 & 2.4 \\
Dimethoate & 0.01 & 0.5 (peppers) & 8 & 5.06 & 3.7 \\
\hline
\end{tabular}

\subsection{Statistical Methods}

In most of the cases pesticide residue dissipation curves can be described mathematically by a first order decay model [32- 38]. Occasionally other mathematical models have to be applied to describe the decay [12]. Results obtained from the analysis of sampled vine leaves were plotted and statistically analysed using the statistical software R (R Core Team, 2017) [39]. In this study, where possible a first order decay model was used to describe the data according to Equation 1:

$$
\mathrm{C}_{\mathrm{t}}=\mathrm{C}_{0} \times \exp \left(-\mathrm{k}_{\text {diss }} \times \mathrm{t}\right)
$$

Where $C_{t}$ is the residual pesticide concentration in the vine leaves $(\mathrm{mg} / \mathrm{kg})$ at time $\mathrm{t}, \mathrm{C}_{\mathrm{o}}$ is the initial pesticide concentration, in $\mathrm{mg} / \mathrm{kg}$ on the harvested plant material immediately after pesticide application, $t$ the time since spraying and $\mathrm{k}_{\mathrm{diss}}$ is the dissipation constant, which is related to the rate of pesticide breakdown. Equation 1 is non-linear and implicitly assumes a constant variance along time. A logarithmic transformation is therefore applied resulting in Equation 2, which is linear:

$$
\operatorname{Ln}\left(\mathrm{C}_{\mathrm{t}}\right)=\ln \left(\mathrm{C}_{0}\right)-\mathrm{k}_{\text {diss }} \times \mathrm{t}
$$

Equation 2, was applied to fit the residue data obtained for all the pesticides. An alternative approach was required for diazinon. In the case of diazinon, a biphasic model was required to obtain homoscedasticity, i.e. homogeneity of variances. This was achieved by minimising the sum of 
squares of the residuals on the logarithmically transformed data. Rearranging Equation 2 and solving for $\mathrm{k}_{\mathrm{diss}}$ yields Equation 3:

$$
\mathrm{k}_{\mathrm{diss}}=\left(\ln \left(\mathrm{C}_{0}\right)-\ln \left(\mathrm{C}_{\mathrm{t}}\right)\right) /(\mathrm{t})
$$

$\mathrm{k}_{\mathrm{diss}}$ represents the slope of the regression line. From this, one can finally obtain the half-life $\left(t_{1 / 2}\right)$, defined as the time needed to reduce the initial pesticide residue level to half of its initial value [33], and calculated from Equation 4:

$$
\mathrm{t}_{1 / 2}=\ln (2) / \mathrm{k}_{\mathrm{diss}}
$$

For each pesticide there were two data series, based on the different study areas. Where these two series had different starting residue levels at the time of the first sampling, a convenient method for estimating the initial application residue level was to use the intercepts of the regressions models for each data series separately. The maximum intercept value was used further to estimate the half-life and the required WHP for that pesticide. The WHP was estimated as the time needed for the residues to dissipate to values equivalent to the MRL after pesticide application (time 0) using the established regression model. The Equation used was:

$$
\mathrm{WHP}=\left[(\text { intercept }-\ln (\mathrm{MRL} \text { value })) / \mathrm{k}_{\text {diss }}\right]
$$

Paramasivam et al. [40] used Equation 5 to calculate the pre-harvest interval (PHI) defined as the maximum time (days) required for the residues to fall below the MRL. Confidence limits (CL) for the regression curves were obtained from Equation 6:

$$
\mathrm{CL}=\mathrm{X} \pm \mathrm{t} \mathrm{SE}
$$

Where $\mathrm{t}$ is the Student's $\mathrm{t}$ value and SE is the standard error. The statistical model used to describe the data included additional terms to take into account the effect of the study areas, the effect of washing and their interactions. Where the results indicated that these terms were not significant, then the model was simplified and those additional terms deleted. Confidence limits for the WHP were obtained from the confidence limits of the regression slope, using a stepwise approach, at the point of intersection between the regression line and the MRL. The first step in estimating the confidence limits for the WHP was to estimate the standard error of the fitted line at the intersection point of the regression line with the MRL value (as shown in Figure 1). Finally, the standard error value (SE) was divided by the slope (with its sign) of the regression line to obtain the SE of the WHP. The confidence interval for the WHP was then obtained from Equation 6. The appraisal of the withholding period required to achieve the MRL value for diazinon was estimated using a two-compartment model following a Newton-Raphson technique [41]. Confidence limits of the required withholding period were estimated for the two-compartment model using a bootstrap technique [42], which was used to obtain 1000 estimates. The quantiles of the estimates obtained were used for the estimation of the confidence limits of the withholding period.

\section{Results and Discussion}

For each of the organophosphate pesticides in this investigation, the residues data obtained from the analytical method were mathematically transformed according to Equation 2 and, where possible, a first order decay model was fitted to the data using the statistical software $\mathrm{R}$ and following the equations above. Table 3 presents a summary of the WHP for the studied pesticides.

Table 3. Comparative summary of the withholding period (WHP) for chlorpyrifos, diazinon and dimethoate according to the Codex and EU MRL. Estimated confidence intervals (in days) are for unwashed leaves. Lower confidence level (LCL) and upper confidence level (UCL).

\begin{tabular}{lllll}
\hline Pesticide & Estimate WHP (Codex) & LCL-UCL & Estimate WHP (EU) & LCL-UCL \\
\hline Chlorpyrifos & 26.0 & $22.2-29.5 ;$ & 37.0 & $31.6-42.1$ \\
Diazinon & 8.9 & $6.0-10.6$ & 37.1 & n/a \\
Dimethoate & 19 & $18-20$ & 33 & $31-34$ \\
\hline
\end{tabular}

\subsection{Chlorpyrifos Methyl}

No detectable residues were found for chlorpyrifos methyl (CPM). None of the samples analysed showed CPM residues above the limit of detection. This behaviour can be explained by photolysis, as shown by the photolysis half-life data in Table 2, and a fast dissipation rate of CPM under the local conditions in Syria. This is in line with previous findings about this pesticide for which the half-life ranged from 0.97 to 3.27 days in Italy [17)] or 0.9-2.6 days on different foliage [7]. The concentration of chlorpyrifos methyl applied at the Al-Hamah site was very small $(200 \mathrm{~mL}$ of $20 \%$ pesticide formulation dissolved in $50 \mathrm{~L}$ of water) compared to that used at the Al Soujah site $(280 \mathrm{~mL}$ of $20 \%$ pesticide dissolved in $7 \mathrm{~L}$ of water). However, there is no information about the authenticity of the pesticide formulation. No statistical analysis could be carried out on the data relating to CPM.

\subsection{Chlorpyrifos}

The details of the regression model of chlorpyrifos presented in Table 4 indicates that there is an effect for the variables time and study area (Al-Hamah and Al Soujah), but there is no interaction between the variables. 
Table 4. Details of the regression model of chlorpyrifos residue data (in natural logarithm scale) for dissipation time and study area.

\begin{tabular}{lllllll}
\hline Parameter & Statistical estimate & Standard Error & t value & Pr $(>|\mathbf{t}|)$ & Lower 95\% & Upper 95\% \\
\hline Time 0 (intercept value) & 4.73 & 0.19 & 24.76 & $<<0.001$ & 4.34 & 5.12 \\
Dissipation time (slope value) & -0.209 & 0.016 & -13.00 & $<<0.001$ & -0.243 & -0.1176 \\
Study area (slope value) & -1.589 & 0.233 & -6.83 & $<<0.001$ & -2.069 & -1.1096 \\
\hline
\end{tabular}

That means it is possible to construct separate regression models for each of the study areas. However, it is acceptable to build a regression model with an average slope from both series [42] as shown in Figure 1A.

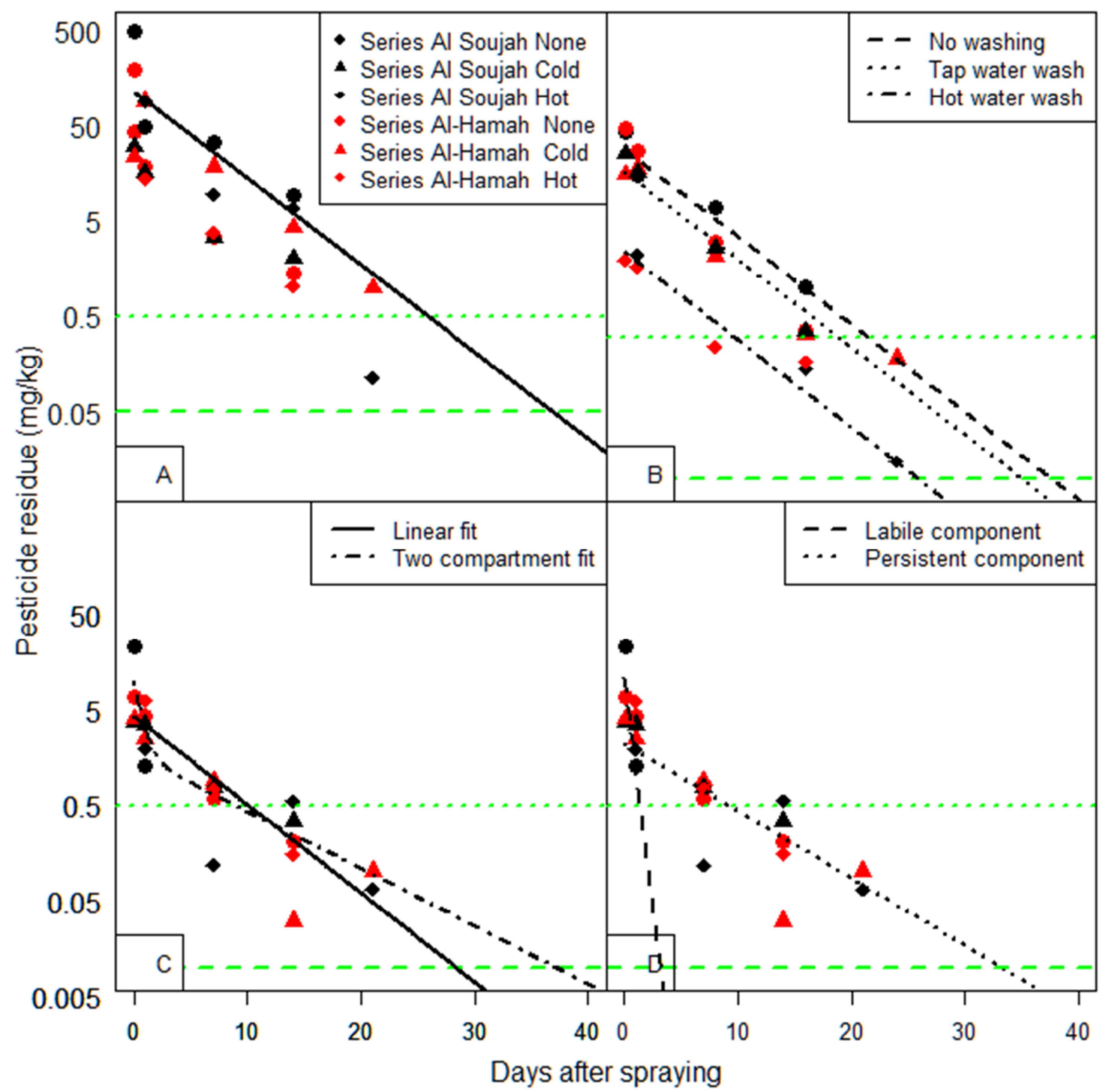

Figure 1. A) logarithmic decay of chlorpyrifos for the estimation of dissipation of chlorpyrifos; the fitted line was based on Al Soujah data; B) logarithmic decay of dimethoate for the estimation of dissipation of dimethoate and showing the effect of washing; C) logarithmic decay of diazinon for the estimation of dissipation of diazinon and showing linear and two compartment models; D) showing the modelled dissipation of the labile and persistent compartments for diazinon.

In this study the first sample of vine leaves was collected only after 60 minutes from pesticide application, due to manpower constraints and other operational factors. The volatile losses from leaves that may have occurred immediately after application can in fact be substantial [43]. Therefore, the initial application residue data, at time 0 hours, was estimated from the intercept of the regression model from the highest residue data set (Al Soujah). This conservative approach was taken to avoid overestimating the half-lives. The intercept (i.e. time zero) was estimated from the constructed models. The initial pesticide residue loads for the two study locations were $114 \mathrm{mg} / \mathrm{kg}$ (Al Soujah series) (back transformed data for 4.73) and $23.22 \mathrm{mg} / \mathrm{kg}$ (back transformed data for 3.14) (Al Hamah series), see Table 4.

Although the initial loads seemed different between the study sites, from a statistical point of view there was no significant difference. The estimated half-life for chlorpyrifos was calculated using Equation 4, which gave an estimate of 
3.3 days. Using the standard error of the slope (0.016), $95 \%$ confidence limits for the half-life were established at $2.9-$ 3.9 days. The estimated half-life was similar for both data sets from each study area. These results for half-life values for chlorpyrifos are in line with previous ranges of 2.9-4 days as reviewed by Willies et al. [7] and $\mathrm{Lu}$ [10] in various foliage crops. The suggested withholding period (WHP) for chlorpyrifos was estimated using Equation 5. A key component of the estimate of the withholding period was the initial load. For chlorpyrifos this varied widely between the two study areas - each estimate having its own confidence limits. Several estimates of the initial load were, therefore, considered, including the average of the estimates, the maximum of the two estimates and the upper confidence limit of the upper estimate. The latter was chosen as a worstcase scenario, providing a safety margin when estimating the WHP. The initial load corresponded to $168.9 \mathrm{mg} / \mathrm{kg}$. Depending on which MRL was used for the calculation, the WHP for chlorpyrifos, i.e. the time required to reduce the initial load of $168.9 \mathrm{mg} / \mathrm{kg}$ to $0.5 \mathrm{mg} / \mathrm{kg}$ if using the Codex MRL, or to $0.05 \mathrm{mg} / \mathrm{kg}$ if using the EU MRL for vine leaves as shown in Table 2, ranged from 26 to 37 days respectively. The WHP for chlorpyrifos is shown in Figure 1A as the time corresponding to the intersection point between the regression line and the MRL. This difference in WHP is significant and implicitly influences the agricultural practices in a relevant way. It also implies that vine leaves should not be used for human or animal consumption for the entire duration of the WHP. Approximate confidence ranges for the withholding period (based on the initial load of $168.9 \mathrm{mg} / \mathrm{kg}$ ) were then 22.2-29.5 days (using Codex MRL) and 31.6-42.1 days (using EU MRL). These confidence limits can only be considered as theoretical extrapolations assuming that the dissipation behaviour of chlorpyrifos approximated a linear function, as samples of vine leaves were not collected at those times. Each collected sample of vine leaves was also washed using tap water and boiling water. In on the case of chlorpyrifos, there was no statistically significant effect of washing the vine leaves with tap water or hot water. There was also no interaction between washing and the effect of dissipation in time. That means that washing the leaves did not decrease the chlorpyrifos load at any of the residue levels studied.

\subsection{Dimethoate}

In the case of dimethoate there was no statistically significant difference between the data sets from the two study areas but there was a large difference between the washing treatments. There was no interaction between the washing treatments and the holding time. Washing with water removed the same fraction of the pesticide over time. Details of the initial estimated loads are given in Table 5.

Table 5. Details of regression of dimethoate against washing type and time.

\begin{tabular}{llllll}
\hline Washing & Estimate $(\mathbf{l n})$ & Std.Error & Estimated initial residue level at t0 $(\mathbf{m g} / \mathbf{k g})$ & t value & Pr $(>\mathbf{F})$ \\
\hline No washing & 3.34 & 0.18 & 28.2 & 18.13 & 15.15 \\
Tap water & 2.79 & 0.18 & 16.30 & 4.62 & $<<0.001$ \\
Hot water & 0.85 & 0.18 & 2.3 & -18.9 & $<<0.001$ \\
Slope & -0.242 & 0.013 & & $<<0.001$ \\
\hline
\end{tabular}

There was no significant difference between the two study areas. One of the results from the hot water wash series in area 1 (Al Soujah) had an initial value comparable to that of the samples with no washing. This is most probably an outlier but was included in the analysis as there was no valid reason for rejecting it. An estimate of the half-life was obtained using Equation 4 which gave an estimate of 2.9 days. By using the standard error of the slope, 95\% confidence limits for the half-life were 2.6 - 3.2 days. These estimated values are in line with previous findings for which the half-lives for dimethoate were in the range of 0.8-7.2 days depending on the crop [43]. The time required to reduce the initial load of $28.2 \mathrm{mg} / \mathrm{kg}$ (back-transformed value of 3.34) to the MRL value was calculated according to Equation 5 and shown in Figure 1B. The WHP for unwashed leaves was estimated as 19 days to accomplish the Codex MRL and 33 days to reach the EU MRLs, with approximate confidence intervals for the WHP as shown in Table 6.

Table 6. Estimated confidence intervals (in days) for withholding period for dimethoate for washed and unwashed leaves. Lower confidence level (LCL) and upper confidence level (UCL).

\begin{tabular}{lllllll}
\hline & With EU-MRL of 0.01 $\mathbf{~ m g} / \mathbf{k g}$ & & \multicolumn{3}{c}{ With Codex Alimentarius-MRL of $\mathbf{0 . 3} \mathbf{~ m g} / \mathbf{k g}$} \\
\hline Washing & LCL & Estimate (days) & UCL & LCL & Estimate (days) & UCL \\
\hline None & 31 & 33 & 34 & 18 & 19 & 20 \\
Tap water & 29 & 31 & 32 & 16 & 17 & 17 \\
Hot water & 22 & 23 & 24 & 8 & 8 & 9 \\
\hline
\end{tabular}

These differences in WHP demonstrate the need to adopt the agricultural practices that best protect consumers and the environment. The MRL is a conventional measure that needs to reflect the conditions of use under specific environmental conditions, hence the need to generate sufficient support data, from all regions in the world, that can help set and harmonize
MRLs in international meetings and bodies such as the Codex Alimentarius Commission. Cabras et al. [44] provided data on the dissipation of dimethoate on grapes, showing that after 8 days from treatment $80 \%$ of dimethoate was dissipated in the field. The final residue then remained constant in the following 3 weeks. As to the effect of 
washing, details of the regression of dimethoate against the washing regimes and holding time (average slope) and the series is given in Table 6. Figure $1 \mathrm{~B}$ shows the effect of washing on the dissipation of dimethoate. There was a very significant effect of hot water washing with an effective reduction of $92 \%$ of the residue level by washing the leaves with hot water. There was also a reduction of the residue level by washing with tap water but that effect was not statistically significant. This result is in line with results presented in an extensive review by Kaushik et al. [28], which demonstrates that washing and cooking help eliminate most of the pesticides in different food commodities.

\subsection{Diazinon}

In the case of diazinon the linear regression model according to Equation 2 fitted the data very poorly (correlation $r^{2}=0.78$ ). Diazinon showed a rapid decomposition phase in the first day ( $\mathrm{s}$ ) followed by a slower decomposition phase (see Figure 1C). This observation and the difference in dissipation kinetics may be explained by the fact that pesticides applied to the vine leaves may be adsorbed, absorbed, altered, volatilized, or removed by water and the integrated results of these processes may be seen as an initial rapid decline in surface residues followed by a slower, asymptotic decrease [36]. Environmental factors, especially sunlight through photolysis, may cause a very rapid dissipation of diazinon within days from pesticide application. In addition, some of the diazinon may be absorbed by the plant tissues, reaching dynamic equilibrium and degrading thereafter very slowly [45].

An alternative two compartment model was therefore used for diazinon, as discussed by Torabi et al. [45]. The model is described by Equation 7, and graphically shown in Figure $1 \mathrm{D}$.

$$
\mathrm{C}_{\mathrm{t}}=\mathrm{C}_{\mathrm{A}} \times \exp \left(-\mathrm{k}_{1} \times \text { time }\right)+\mathrm{C}_{\mathrm{B}} \times \exp \left(-\mathrm{k}_{2} \times \text { time }\right)
$$

Where $C_{A}$ was the initial amount of rapid phase (estimated at $t=0)$ and $k_{1}$ the dissipation rate in the rapid dissipation phase and $\mathrm{C}_{\mathrm{B}}$ the amount in the slow phase (estimated at time $\mathrm{t}=$ change of slope) and $k_{2}$ the dissipation rate in the slow dissipation (persistent) phase. The parameter estimates for Equation 7 are shown in Table 7.

Table 7. Details of linear regression of diazinon residue data (in natural logarithm scale) against dissipation time for fast and slow phases.

\begin{tabular}{lllll}
\hline \multirow{2}{*}{ Phase } & Intercept & \multicolumn{3}{c}{ Slope } \\
\cline { 2 - 5 } & Estimate & Std. Error & Estimate & Std. Error \\
\hline Fast & 2.109 & 0.311 & -1.043 & 0.420 \\
Slow & 0.296 & 0.533 & -0.135 & 0.039 \\
\hline
\end{tabular}

The slope of the regression line is derived by taking the differential from Equation 7 and estimated as shown by Equation 8:

$$
\mathrm{K}_{\text {diss }}(\text { Slope })=-\mathrm{CA} \times \mathrm{k}_{1} \times \exp \left(-\mathrm{k}_{1} \times \mathrm{t}\right)-\mathrm{CB} \times \mathrm{k} 2 \times \exp \left(-\mathrm{k}_{2} \times \mathrm{t}\right)
$$

The estimated half-life of diazinon varies with time since the initial application of pesticides as shown in Figure 2.

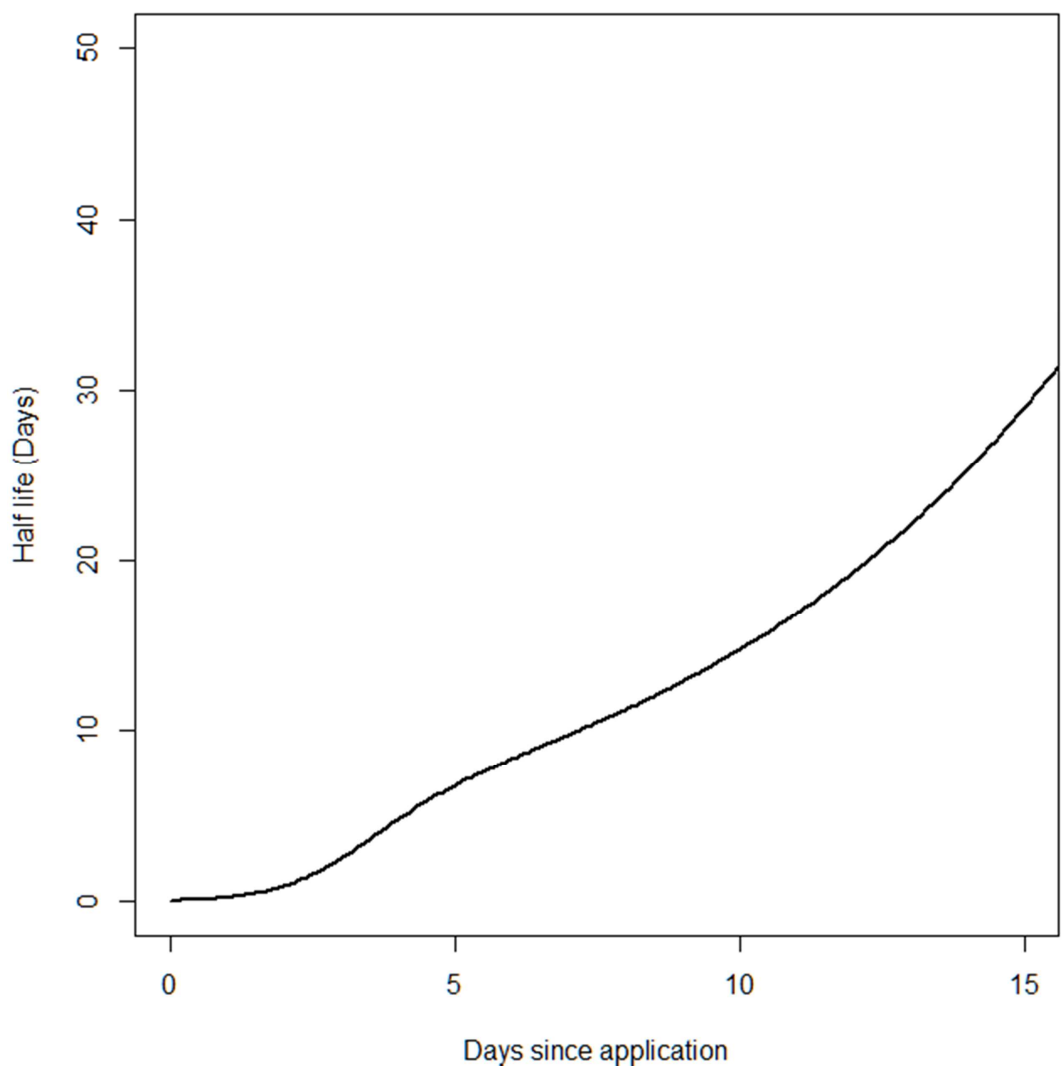

Figure 2. Changes in estimated half-life for diazinon with time since pesticide application. 
These results are in line with previous findings by Torabi et al. [45], Willis et al. [43], where half-lives data for diazinon were in the range of 0.4-5.3 days depending on the crop. The estimation of the initial load of diazinon at time 0 (application time) was estimated in two different ways. A direct method was to sum the $\mathrm{C}_{\mathrm{A}}$ and $\mathrm{C}_{\mathrm{B}}$ terms from Equation 7 (Table 8) and this gave an estimate of $10.06 \mathrm{mg} / \mathrm{kg}$.

Table 8. Details of two compartmental model for diazinon residue data. Indication of the regression estimates for the slow component.

\begin{tabular}{lll}
\hline Parameter & Estimate $(\mathbf{m g} / \mathbf{k g})$ & Std. Error \\
\hline CA & 8.34 & 2.99 \\
CB & 1.72 & 2.61 \\
k1 & 1.53 & 1.01 \\
k2 & 0.139 & 0.16 \\
\hline
\end{tabular}

An alternative method was to use the linear approximation, using the sum of the back-transformed intercepts, as shown in Table 7, which gave an estimation of $9.58 \mathrm{mg} / \mathrm{kg}$. The two values are not significantly different. The WHP was then estimated based on the twocompartment model using the slow component. This gave an estimate, using the Codex MRL, of 8.9 days with confidence intervals of 6.0-10.6 days. The estimated time to achieve the EU's MRL was 37.1 days. The latter value is outside the range of the sampling time and is therefore considered as an approximation only, and no confidence limits can be provided. It is important that estimates of WHP are based on residue data from a sampling regime that includes the estimated time as one cannot be sure that the behaviour of the pesticides in the field follows a linear trend. In relation to the washing of the leaves for diazinon, there was no statistically significant effect of washing the vine leaves with tap water or hot water. There was also no interaction between washing and the effect of time. Although the difference was not statistically significant, the no wash treatment had more pesticide, about 30\% more than the cold wash. Kaushik et al. [28] reviewed food processing treatments such as washing and cooking, among others, and demonstrated that in most cases washing and cooking particularly lead to large reductions in pesticide residue levels. Angioni et al. [46] demonstrated that washing with tap water reduced the residues of azoxystrobyn and fenhexamid but not pyrimethanil. Similarly, in relation to washing, the three pesticides for which data were collected in this study behaved in very different ways. For better comprehension of the observed behaviour, Table 2 presents selected properties of the three studied pesticides. Chlorpyrifos is not a systemic insecticide [1], however it has a high Kow/Koc and would have been strongly bound to the cuticles of the leaves, so there would have been very little wash-off. This is in line with studies by Teixerira et al. [47] and Buschhaus et al. [48] on the composition of the epicuticular composition of leaves and their water barrier action as well as the mobility of pesticides in the plant where lipophilicity and concentration of the active ingredient are the driving forces in the transfer. Adjuvants in commercial formulations may also regulate the transfer processes. Ling et al. [49] came to similar conclusions for this pesticide. Dimethoate has a low Kow/Koc and hence the wash-off was effective - especially when hot water was used. The dissipation of dimethoate in time, and especially the persistent portion, may be explained by translocation of the residues into other plant compartments, since it is classified as a systemic pesticide [47]. This is also in line with studies by Cabras et al. [50], where he showed that dimethoate degraded rapidly during the first week after pesticide application but remained constant in the following two weeks. The intermediate Kow/Koc values for diazinon may have resulted in a fraction of the pesticide being gradually absorbed into a protected layer, i.e. the epicuticular wax of the leaves, which progressively increases in terms of yield during the surface development of the vine leaves as suggested by Buschhaus et al. [48] and previously by Baker et al. [51], Torabi et al. [45], Willis et al. [7], Lu et al [10]. The results from the present study agree with previous data for OPs. Willis et al. [7] reported average values of half-life for OPs of $3.0 \pm 2.7$ days for foliage. Cabras et al. [50] reported half-lives for OPs in grapes, wine and their processing products ranging between 0.97 and 3.84 days. Angioni et al. [22] showed decline curves for boscalid that do not follow a linear decay curve and stressed the idea that field agricultural practices critically influence the decrease or the disappearance of pesticide residues. Fantke et al. [13] provided impressive information by reviewing 811 publications in the scientific literature. Comparative assessment showed that that $95 \%$ of all half-lives, for all pesticide-plant species combinations, fall within the range of 0.6 and 29 days. Marin et al. [14] found that dissipation rates for selected pesticides (especially cyprodinil) were higher in the field at ambient temperature and with natural light than in cold conditions and in darkness. Chemical degradation caused by high temperatures and solar radiation is an important factor in pesticide dissipation kinetics. In the study presented here, environmental conditions possibly favoured chemical degradation in the field, which would have contributed to the short half-life of the studied OP pesticides.

\section{Conclusions}

Vine leave samples were collected over a 21-day period and analysed to assess the decay of four OPs, chlorpyriphosmethyl, chlorpyriphos, diazinon and dimethoate. Analysis of the data provided an indication of the dissipation rates, the half-lives and the WHPs. The three pesticides that could be evaluated behaved in very different ways. For diazinon, regression analysis could not fit the dissipation data to a first order decay model. The estimate of the half-life and withholding time for diazinon depended on the model used. The results from the present study are in line with previous data for organophosphorus pesticides. As part of the study 
vine leaves were also washed with cold and boiling tap water. The data indicate that washing with hot water removed approximately $92 \%$ of the dimethoate residues. This offers a practical method of decreasing the intake of that pesticide and directly protects consumers of, for example, stuffed leaves. To conclude, the reported study can be replicated and applied in field monitoring, with the objective of gaining information on the results of current agricultural practices, thus contributing to set MRLs under the conditions of use in countries worldwide.

\section{Acknowledgements}

This work was supported by IAEA technical cooperation project $\mathrm{SYR} / 5 / 023$.

\section{Appendix}

The study areas are presented in Figures A1 and A2. They are Al Soujah (Yafour) area, located $23 \mathrm{~km}$ west of Damascus, and the Al-Hamah area located $14 \mathrm{~km}$ north west of Damascus respectively.

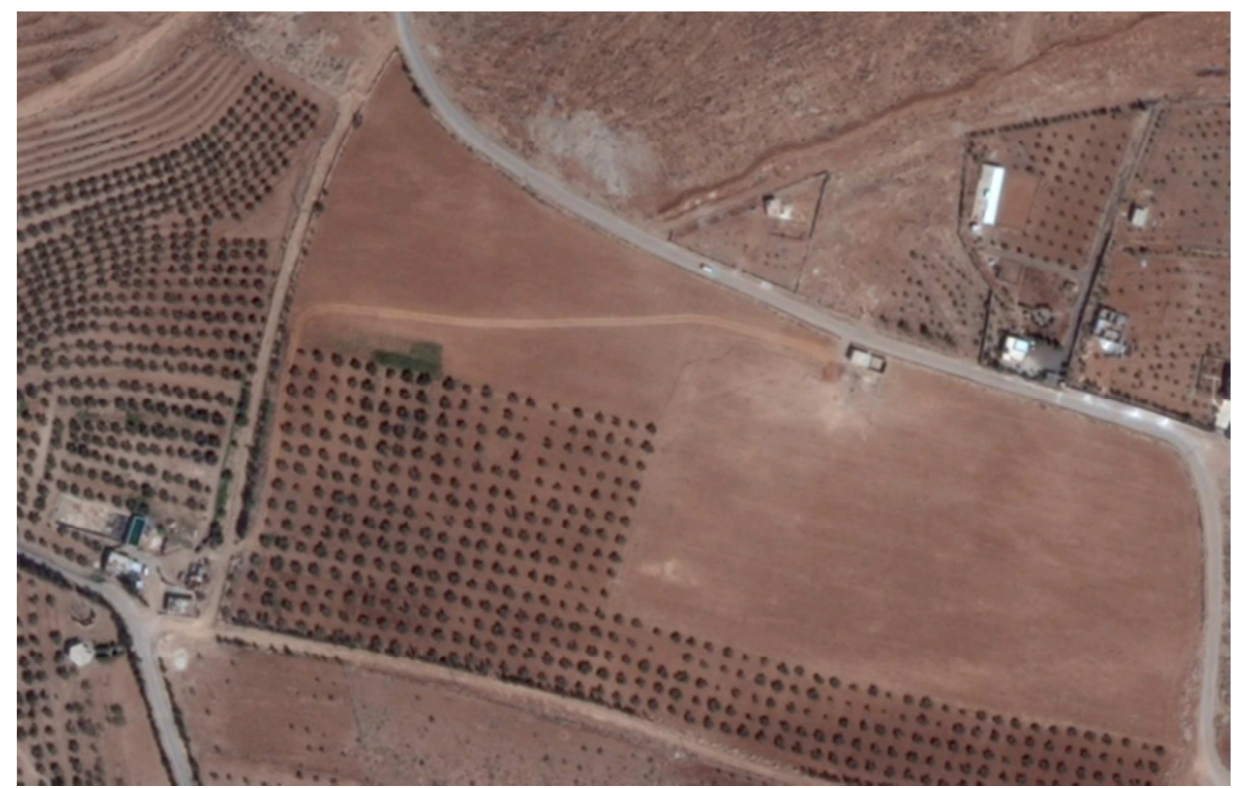

Figure A1. Al Soujah area. Vineyard surrounded by olive trees. Located west of Yafour.

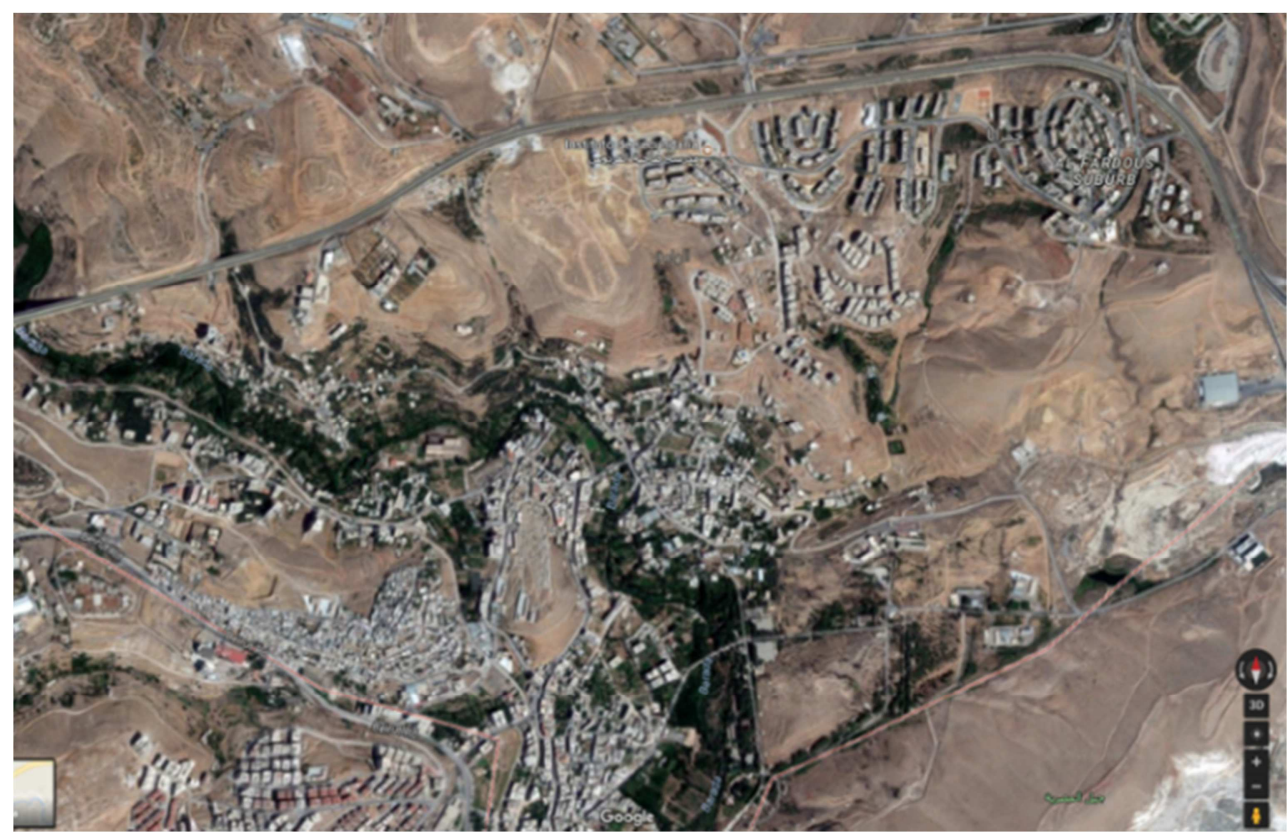

Figure A2. Al Hamah area, a river crosses the area.

Table A1 gives the list of the pesticides, their retention times, the transitions used for quantitation and confirmation together with the optimised collision energies (CE) and the corresponding retention time windows in the multiple reaction monitoring (MRM) acquisition method obtained using a gas chromatograph coupled to tandem mass spectrometry (GC-MS/MS). 
Table A1. List of pesticides, their retention times, the transitions used for quantitation and confirmation together with the optimised collision energies (CE) and the corresponding retention time windows in the multiple reaction monitoring (MRM) acquisition method.

\begin{tabular}{|c|c|c|c|c|c|c|}
\hline Pesticides & MRM Time segment & RT (min) & Quantifier transition 1 & CE $1(V)$ & Qualifier transition 2 & CE2 (V) \\
\hline Chlorpyrifos & 11 & 12.0 & $196.9->169.0$ & 15 & $198.9->171.0$ & 15 \\
\hline Chlorpyrifos-methyl & 8 & 10.8 & $285.9->92.9$ & 25 & $287.9->92.9$ & 25 \\
\hline Diazinon & 6 & 9.5 & $137.1->84.0$ & 10 & $137.1->54.0$ & 20 \\
\hline $\begin{array}{l}\text { Dimethoate } \\
\text { Internal standards }\end{array}$ & 4 & 8.6 & $86.9->46.0$ & 15 & $92.9->63.0$ & 10 \\
\hline Sulfotep & 3 & 8.0 & $201.8->145.9$ & 10 & $201.8->81.9$ & 25 \\
\hline Triphenyl phosphate & 19 & 15.3 & $326.0->325.0$ & 5 & $214.9->168.1$ & 15 \\
\hline
\end{tabular}

Method validation information for each pesticide including method limit of quantitation (LOQ), and the precision data obtained during method validation are presented in Table A2. The limit of quantitation (LOQ) of the method was the lowest fortification level of the validation meeting the method performance acceptability criteria and it was obtained from recovery studies.

Table A2. Method validation information for each pesticide including method limit of quantitation (LOQ), average recovery and intermediate precision data (repeatability and reproducibility relative standard deviation) at $0.01 \mathrm{mg} / \mathrm{kg} \mathrm{level}$.

\begin{tabular}{lllll}
\hline Pesticide & LOQ (mg/kg) & Average recovery (\%) & Repeatability RSD (\%) at 0.01 mg/kg & Reproducibility RSD (\%) at 0.01 mg/kg \\
\hline Chlorpyrifos & 0.01 & 83 & 15 & 16 \\
Chlorpyrifos-methyl & 0.01 & 81 & 13 & 15 \\
Diazinon & 0.01 & 82 & 13 & 14 \\
Dimethoate & 0.01 & 81 & 9 & 9 \\
\hline
\end{tabular}

\section{References}

[1] Food and Agriculture Organization. FAO specifications and evaluations for agricultural pesticides 2018. www.fao.org/agriculture/crops/thematic-

sitemap/theme/pests/jmps/ps-new/en/\#D (accessed Jan 2018).

[2] Maia, M.; Monteiro, F.; Sebastiana, M.; Marques, A. P.; Ferreira, A. E. N.; Freire, A. P.; Cordeiro, C.; Figueiredo, A.; Silva, M. S. Metabolite extraction for high-throughput FTICR-MS-based metabolomics of grapevine leaves. EuPA Open Proteomics 2016, 12, 4-9.

[3] Tsiropoulos, N. G.; Miliadis, G. E; Likas, D. T.; Liapis K. Residues of spiroxamine in grapes following field application and their fate from vine to wine. J Agric Food Chem. 2005, 53 (26), 10091-6.

[4] Grimalt, S.; Dehouck, P. Review of analytical methods for the determination of pesticide residues in grapes. J Chromatogr A 2016, 12, 1433, 1-23.

[5] Del Carlo, M.; Mascini, M.; Pepe, A.; Compagnone, D.; Mascini, M. Electrochemical Bioassay for the Investigation of Chlorpyrifos-methyl in Vine Samples. J. Agric. Food Chem. 2002, 50 (25), 7206-7210.

[6] Arora, P. K; Jyot, G.; Singh, B.; Singh Battu, R.; Singh, B.; Singh Aulakh, P. Persistence of Imidacloprid on Grape Leaves, Grape Berries and Soil. Bull. Environ. Contam. Toxicol. 2009, 82, 239-242.

[7] Willis, G. H.; Mc Dowell, L. L. Pesticide persistence on foliage. Reviews of environmental contamination and toxicology 1987, 100, 23-73.

[8] Pihlström, T. Analysis of pesticide residues in food using ethyl acetate extraction and detection with GC-MS/MS and LCMS/MS (SweET). In Proceedings of the 5th Latin American Pesticide Residues Workshop, food and environment, Cortes,
E.; Gras, N.; Navarrete; D.; Lillo, P., Eds.; Pontificia Universidad Católica de Chile: Santiago de Chile, Chile, 2015; 72 pp.

[9] Montasser, M. R.; Mahmoud, H. A. Alexandria Science Exchange Journal 2009. www.alexexch.org/File/2009003001/En/2087.pdf (accessed Jun 2018).

[10] Lu, M.-X., Jiang, W. W., Wang, J.-L., Liu, X.-J., Yu, X.-Y. Persistence and dissipation of chlorpyrifos in brassica chinensis, lettuce, celery, asparagus lettuce, eggplant, and pepper in a greenhouse. PLoS ONE, 2014, doi: 10.1371/journal.pone.0100556.

[11] Food and Agriculture Organization and World Health Organization. Pesticide residues in food, 2016. www.fao.org/3/a-i5693e.pdf (accessed Jun 2018).

[12] World Health Organization. JMPR toxicological monographs, $2017 . \quad$ www.who.int/foodsafety/publications/jmprmonographs/en/ (accessed Jun 2018).

[13] Fantke, P.; Juraske, R. Variability of pesticides dissipation half-lives in plants. Environ. Sci. Technol. 2013, 47, 35483562 .

[14] Marin, A; Oliva, J; Garcia, C; Navarro, S; Barba, A. Dissipation rates of cyprodinil and fludioxonil in lettuce and table grape in the field and under cold storage conditions. J Agric Food Chem. 2003, 51 (16), 4708-11.

[15] Abdelraheem, E., Arief, M., Mohammad, S., G., Jiang, W. A Safety assessment of chromafenozide residue level with decline study on tomato in Egypt. Environ Monit Assess., 2017, 189, 4, pp.180. doi: 10.1007/s10661-017-5894-6.

[16] Hongfang, L., Xinze, L., Yecheng, M., Kyongjin, P., Jiye, H.. Residue analysis and dietary exposure risk assessment of tebufenozide in stem lettuce (Lactuca sativa L. var. angustana Irish), Food and Chemical Toxicology, 2018, 120, pp. 64-70, doi.org/10.1016/j.fct.2018.06.057. 
[17] Jie, L., Muhammad, R., Jiangwei, Q., Meiying, H., Guohua, Z. Dissipation and metabolism of tebufenozide in cabbage and soil under open field conditions in South China, Ecotoxicology and Environmental Safety, 2016, 134, pp.204212, doi.org/10.1016/j.ecoenv.2016.09.002.

[18] Xiaoxin, C., Sheng, H., Yimen, G., Yecheng, M., Jiye, H., Xiao, L. Dissipation behavior, residue distribution and dietary risk assessment of field-incurred boscalid and pyraclostrobin in grape and grape field soil via MWCNTs-based QuEChERS using an RRLC-QqQ-MS/MS technique. Food Chemistry, 2018. doi.org/10.1016/j.foodchem.2018.08.136.

[19] Soliman, A., S., Helmy, R., M., A., Nasr, I., N., Mahmoud, H., A., Jiang, W. Behavior of Thiophanate Methyl and Propiconazole in Grape and Mango Fruits Under the Egyptian Field Conditions. Bull Environ Contam Toxicol., 2017, 98, 5, pp.720-725. doi: 10.1007/s00128-017-2066-x.

[20] Liu J, Rashid M, Qi J, Hu M, Zhong G. Dissipation and metabolism of tebufenozide in cabbage and soil under open field conditions in South China. Ecotoxicol Environ Saf., 2016, 134, pp. 204-212. doi:10.1016/j.ecoenv.2016.09.002.

[21] Besil, N.; Pérez-Parada, A.; Cesio, V.; Varela, P.; Rivas, F., Heinzen H. Degradation of imazalil, orthophenylphenol and pyrimethanil in Clementine mandarins under conventional postharvest industrial conditions at $4^{\circ} \mathrm{C}$. Food Chem. 2016, 1 (194), 1132-7.

[22] Angioni; A.; Dedola; F.; Garau; V. L.; Schirra; M.; Caboni; P. Fate of iprovalicarb, indoxacarb, and boscalid residues in grapes and wine by GC-ITMS analysis. J Agric Food Chem. 2011, 59 (12), 6806-12.

[23] Cabras, P.; Garau, V., L.; Filippo, M.; Pirisi, F. M.; Cubeddu, M.; Cabitza, F.; Spanedda, L. Fate of some insecticides from vine to wine. J. Agric. Food Chem. 1995, 43 (10), 2613-2615.

[24] Navarro, S.; Oliva, J.; Navarro, G.; and Barba, A. Dissipation of chlorpyrifos, fenarimol, mancozeb, metalaxyl, penconazole, and vinclozolin in grapes. American Journal of Enology and Viticulture 2001, 52, 35-40.

[25] Cus, F.; Basa Cesnik, H.; Bolta, S. V.; Gregorcic, A. Pesticide residues in grapes and during vinification process, Food Control 2010, 21 (11), 1512-1518.

[26] Australian Government. Agricultural and Veterinary Chemicals Code Act 1994, latest edition 2016. www.legislation.gov.au/Series/C2004A04723 (accessed Jun 2018).

[27] Amvrazi, E. G. Fate of Pesticide Residues on Raw Agricultural Crops after Postharvest Storage and Food Processing to Edible Portions. 2011. www.cdn.intechopen.com/pdfs/13027/InTech-

Fate_of_pesticide_residues_on_raw_agricultural_crops_after postharvest_storage_and_food_processing_to_edible_portions .pdf (accessed June $\overline{2} 018)$.

[28] Kaushik, G.; Satya, S.; Naik, S. N. Food processing a tool to pesticide residue dissipation - A review. Food Res. Int. 2009, $42,26-40$.

[29] Holland P. T., Hamilton D., Ohlin B., Skidmore M. W. Effects of storage and processing on pesticide residues in plant products. Pure Appl. Chem. 1994, 66, 335-356.

[30] Maestroni, B.; Alnaser, A. Abu; Ghanem. I.; Islam, M.; Cesio, V.; Heinzen, H.; Kelly, S.; and Cannavan, A. Validation of an analytical method for the determination of selected pesticide residues in vine leaves by GC-MS/MS. J. Agric. Food Chem. 2018, DOI: 10.1021/acs.jafc.8b00453.

[31] Lewis, K.; and Tzilivakis, J. Development of a data set of pesticide dissipation rates in/on various plant matrices for the Pesticide Properties Data Base (PPDB). Data 2017, 2 (3), 28.

[32] Galietta, G.; Egana, E.; Gemelli, F.; Maeso, D.; Casco, N.; Conde, P.; Nunez, S. Pesticide dissipation curves in peach, pear and tomato crops in Uruguay. J Environ Sci Health B. 2011, 46 (1), 35-40.

[33] Yajie, C., Mingcheng, G., Xingang, L., Jun, X., Fengshou, D., Xiaohu, W., Baotong, L., Yongquan, Z. Determination and dissipation of afidopyropen and its metabolite in wheat and soil using QuEChERS-UHPLC-MS/MS. J. Sep. Science, 2018, 41, 7, pp. 1674-1681, doi.org/10.1002/jssc.201700773.

[34] Xiaokang, A., Jun, X., Fengshou, D., Xingang, L., Xiaohu, W., Ran, W., Yongquan, Z. Simultaneous determination of broflanilide and its metabolites in five typical Chinese soils by a modified quick, easy, cheap, effective, rugged, and safe method with ultrahigh - performance liquid chromatography and tandem mass spectrometry, J. Sep. Science, 2018, doi.org/10.1002/jssc.201800631.

[35] Huan, Z., Luo, J., Xu, Z., Xie, D. Residues, dissipation, and risk assessment of spinosad in cowpea under open field conditions. Environ. Monit. Assess. 2015, 187, 11, pp. 706. doi: 10.1007/s10661-015-4942-3.

[36] Saber, A., N, Malhat, F., M, Badawy, H., M, Barakat, D., A. Dissipation dynamic, residue distribution and processing factor of hexythiazox in strawberry fruits under open field condition. Food Chem. 2016, 196, pp. 1108-1116. doi:10.1016/j.foodchem.2015.10.052.

[37] Malhat, F., Badawy, H., M,. A., Barakat, D,. A., Saber, A., N. Residues, dissipation and safety evaluation of chromafenozide in strawberry under open field conditions. Food Chemistry, 2014, 152, pp. 18-22. doi: 10.1016/j.foodchem.2013.11.110.

[38] Malhat, F., El-Mesallamy, A., Assy, M., Madian, W., Loutfy, N., M., Tawfic, M., A. Residues, half-life times, dissipation, and safety evaluation of the acaricide fenpyroximate applied on grapes, Toxicological \& Environmental Chemistry, 2013, 95, 8, 1309-1317, DOI: 10.1080/02772248.2013.877245.

[39] R Core Team, 2017.https://www.r-project.org/ (accessed Jun 2018).

[40] Paramasivam, M.; Deepa, M; Selvi, C.; and Chandrasekaran, S. Dissipation kinetics and safety evaluation of tebuconazole and trifloxystrobin in tea under tropical field conditions. Food Additives \& Contaminants: Part A 2017, 34 (12), 2155-2163.

[41] Upton, G., Cook, I. Oxford Dictionary of Statistics (3rd Edition) Oxford Univ. Press: Oxford, UK, 2014.

[42] Efron, B.; Tibshirani, R. An Introduction to the Bootstrap. Chapman and Hall / CRC: New York, 1994.

[43] Willis, G. H.; Mc Dowell, L. L.; Southwick, L. M.; Smith, S. Toxaphene, methyl parathion, and fenvalerate disappearance from cotton foliage in the Mid South. J. Environ. Qual. 1985, $14,446-450$.

[44] Cabras, P.; Garau, V. L.; Melis, M.; Pirisi, F. M.; Cubeddu, M.; Cabitza F. Residui di dimetoato e chlorpirifos nell'uva e nel vino. Atti Giornate Fitopatologiche 1994, 1, 27-32. 
[45] Torabi, E.; Talebi, K. Diazinon residues and degradation kinetics for grapes under field conditions. J Environ Sci Health B. 2013, 48 (4), 260-5

[46] Angioni, A.; Schirra, M.; Garau, V. L.; Melis, M.; Tuberoso, C. I. G.; Cabras, P. Residues of azoxystrobin, fenhexamid and pyrimethanil in strawberry following field treatments and the effect of domestic washing. Food Addit. Contam. 2004, 21, $1065-1070$

[47] Teixerira, M.; Aguiar, A.; Afonso, C.; Alves, A.; Bastos, M. Comparison of pesticides levels in grape skin and in the whole grape by a new liquid chromatographic multiresidue methodology. Analytica Chimica Acta. 2004, 513, 333-340.

[48] Buschhaus, C.; and Jetter, R. Composition and Physiological
Function of the Wax Layers Coating Arabidopsis Leaves: $\beta$ Amyrin Negatively Affects the Intracuticular Water Barrier. Plant Physiology 2012, 160 (2), 1120-1129.

[49] Ling, Y.; Wang, H.; Yong, W.; Zhang, F.; Sun, L.; Yang, M. L.; Wu, Y. N.; Chu, X. G. The effects of washing and cooking on chlorpyrifos and its toxic metabolites in vegetables. Food Control 2011, 22, 54-58.

[50] Cabras, P.; and Angioni, A. Pesticide Residues in Grapes, Wine, and Their Processing Products. J. Agric. Food Chem. 2000, 48 (4), 967-973.

[51] Baker, E. A.; Hunt, G. M. Developmental changes in leaf epicuticular waxes in relation to foliar penetration. New Phytologist 1981, 88, 731-747. 